\title{
EFFECT OF RADIAL FLOW AND RESONANCE DECAY ON THE TRANSVERSE MOMENTUM DEPENDENCE OF THE HBT PARAMETERS
}

\author{
Yao Zhang ${ }^{\mathrm{a}}$, Anqi Gu ${ }^{\mathrm{b}}$, Desheng $\mathrm{LiU}^{\mathrm{a}}$

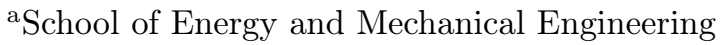 \\ Jiangxi University of Science and Technology \\ Nanchang 330013, China \\ ${ }^{\mathrm{b}}$ School of Computer Science and Information Engineering \\ Harbin Normal University \\ Harbin 150025, China
}

(Received June 8, 2017; accepted November 11, 2017)

Using the Blast-Wave parameterized model in THERMINATOR 2, we study the effect of the radial flow and resonance decay on the transverse momentum dependence of $\mathrm{HBT}$ parameters for $\mathrm{Au}+\mathrm{Au}$ central collisions at the RHIC energy $\sqrt{s_{N N}}=200 \mathrm{GeV}$. The results indicate that the radial flow directly affects the space-momentum correlations and the dependence of the transverse HBT radii on transverse momentum. The rise of $\alpha$ with increasing $v_{\mathrm{T}}$ for $R_{\text {out }}$ is faster than that for $R_{\text {side }}$ which suggests that $R_{\text {out }}$ is more susceptible to the radial flow. The pions decayed from resonance increase the HBT radii and strengthen the negative dependence of the radii on transverse momentum.

DOI:10.5506/APhysPolB.49.5

\section{Introduction}

Femtoscopy measurements (often called HBT analysis) provide the detailed information on the space-time geometry and dynamics of the interaction regions from the system formed in relativistic heavy-ion collisions [1, 2]. The HBT radii are not equal to the real space size of the source directly, but to a 'region of homogeneity', which allows to extract dynamical information of the particle emission source through the transverse momentum dependence of the HBT parameters [3-5]. A drop of the HBT radii with increasing transverse momentum is observed in the experiments of the heavy-ion collisions and the dependence of the radii is similar for all collision energies, which is directly affected by the space-momentum correlations [3, 6, 7]. 
It is generally recognized that the space-momentum correlations of the particles are mainly caused by the collective expansion which is embodied in the transverse flow velocity. The effect of the collective flow on the directly freeze-out pions is different from that on the pions decayed from resonance such as $\rho$ and $\omega[8,9]$. The short-lived resonances increase the HBT radii by about $1 \mathrm{fm}$, which is caused by the resonance lifetime of $1 \mathrm{fm} / c[8,10]$.

For studying the effect of the radial flow and resonance decay on the HBT parameters, we use the THERMal heavy IoN generATOR 2 (THERMINATOR 2) model, a Monte Carlo event generator, to generate the simulated events for $\mathrm{Au}+\mathrm{Au}$ central collisions at the RHIC energy $\sqrt{s_{N N}}=$ $200 \mathrm{GeV}$ [11]. Several freeze-out models can be selected in the THERMINATOR model, such as the Blast-Wave model or the Kraków Single Freeze-out model. The Blast-Wave model is based on the Monte-Carlo method, which generates the stable particles and resonances on a freeze-out hypersurface and then the resonances decay by a cascade approach. The velocity profile of the radial flow can be specified in the Blast-Wave model, which is convenient for studying the effect of the different transverse flow velocities on the transverse momentum dependence of the HBT radii and space-momentum correlations. The Blast-Wave parameterized model is used in the THERMINATOR 2 with a linear transverse flow, $v_{\mathrm{T}}(\rho)=v_{\mathrm{T}}\left(\rho / \rho_{\max }\right)$ and $\rho_{\max }=7.43 \mathrm{fm}$. The transverse flow velocities are set at $v_{\mathrm{T}}=0,0.2,0.4,0.6 c$ to compare the transverse momentum dependence of the HBT radii, respectively.

\section{The transverse momentum dependence of the HBT parameters}

The two-pion correlation function is calculated by the Correlation After Burner (CRAB), without the final-state interactions in the program [12]. For central collisions, the HBT correlation function is often fitted by the Bertsch-Pratt parametrization in the 'out-side-long' coordinate system [13]

$$
C(\boldsymbol{q}, \boldsymbol{K})=1+\lambda \exp \left(-q_{\text {out }}^{2} R_{\text {out }}^{2}(\boldsymbol{K})-q_{\text {side }}^{2} R_{\text {side }}^{2}(\boldsymbol{K})-q_{\text {long }}^{2} R_{\text {long }}^{2}(\boldsymbol{K})\right),
$$

where $\boldsymbol{q}=\boldsymbol{p}_{\mathbf{1}}-\boldsymbol{p}_{\mathbf{2}}, \boldsymbol{K}=\left(\boldsymbol{p}_{\mathbf{1}}+\boldsymbol{p}_{\mathbf{2}}\right) / 2$, and $q_{\text {out }}, q_{\text {side }}, q_{\text {long }}$ are three components of the relative momentum difference $\boldsymbol{q}$ of a pair of pions which are defined in the 'out-side-long' system. By fitting the correlation function, we can obtain size parameters $R_{\text {out }}, R_{\text {side }}$ and $R_{\text {long }}$ which are so-called HBT radii.

Figure 1 shows the pion HBT radii $R_{\text {out }}, R_{\text {side }}, R_{\text {long }}$, the ratio $R_{\text {out }} / R_{\text {side }}$ and $\lambda$ parameter as a function of the pair average transverse mass $m_{\mathrm{T}}$ in central $\mathrm{Au}+\mathrm{Au}$ collisions at $\sqrt{s_{N N}}=200 \mathrm{GeV}$ with the transverse flow velocity $v_{\mathrm{T}}=0,0.2,0.4,0.6 c$, where $m_{\mathrm{T}}=\sqrt{\boldsymbol{K}^{2}+m_{\pi}^{2}}$ and $m_{\pi}$ is the mass of pion. $v_{\mathrm{T}}$ reduces the values of radii $R_{\text {out }}$, increases the values of 

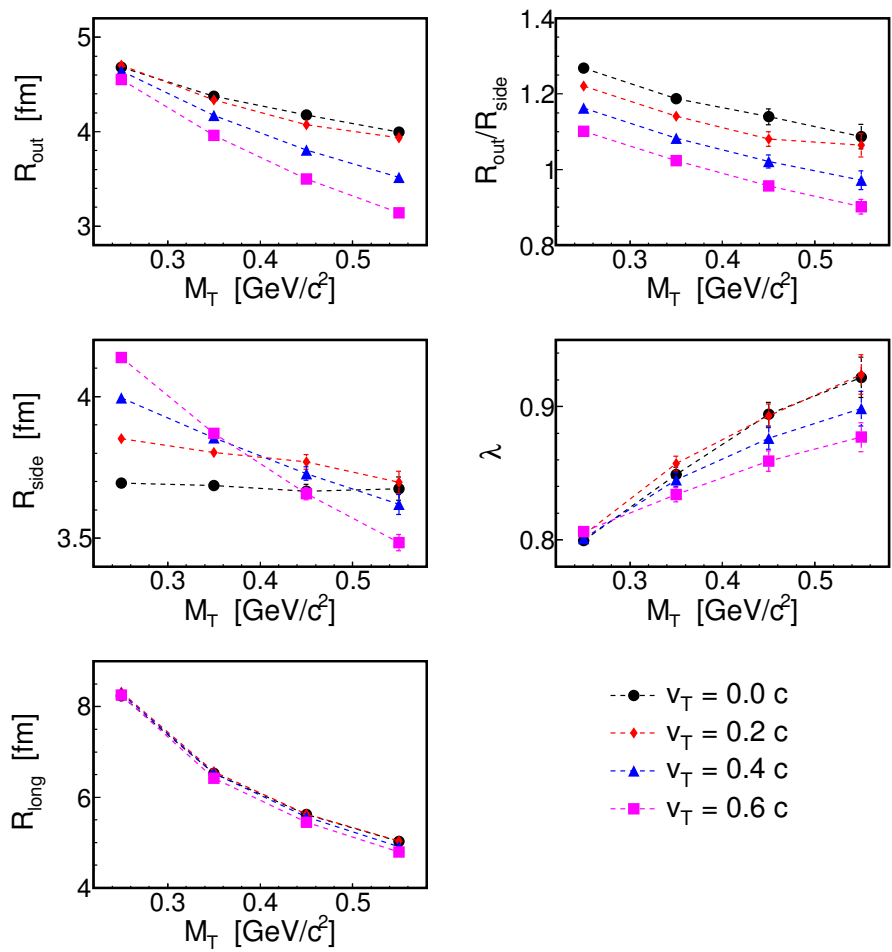

$-\mathrm{v}_{\mathrm{T}}=0.0 \mathrm{C}$

$\cdots \mathrm{v}_{\mathrm{T}}=0.2 \mathrm{c}$

$-\cdots-v_{T}=0.4 \mathrm{C}$

$-v_{\mathrm{T}}=0.6 \mathrm{c}$

Fig. 1. The HBT parameters at mid-rapidity as functions of $m_{\mathrm{T}}$ with different $v_{\mathrm{T}}$ for $\mathrm{Au}+\mathrm{Au}$ central collisions at the RHIC energy of $\sqrt{s_{N N}}=200 \mathrm{GeV}$.

radii $R_{\text {side, }}$ and does not affect the radii $R_{\text {long. }}$. The radii $R_{\text {out }}$ and $R_{\text {side }}$ decrease with $m_{\mathrm{T}}$ and the decrease is now rapid as $v_{\mathrm{T}}$ increases. The value of $v_{\mathrm{T}}$ does not affect the drop of $R_{\text {long }}$. When $v_{\mathrm{T}}$ is set to zero, $R_{\text {side }}$ remains unchanged as $m_{\mathrm{T}}$ increases and $R_{\text {out }}$ decreases with increasing $m_{\mathrm{T}}$, which indicates the drop of $R_{\text {side }}$ is caused by the radial flow and the transverse momentum dependence of $R_{\text {side }}$ is not only affected by the radial flow, but also by the other space-momentum correlations [5, 15].

Figure 2 shows the HBT radii with different $v_{\mathrm{T}}$ and the fitted results using the power-law function $R_{i}\left(m_{\mathrm{T}}\right)=R_{i}^{\prime}\left(m_{\pi} / m_{\mathrm{T}}\right)^{\alpha}$. The power-law fit is derived by the ideal hydrodynamical simulation, in which $\alpha=0.5$ [16]. It is obvious that the fitted results are close to the HBT radii, which indicates that the power-law function is appropriate to fitting the HBT radii.

Figure 3 shows the extracted parameters $R^{\prime}$ and $\alpha$ by the power-law fit as a function of the transverse velocities, where solid lines and dashed lines show the results of the pions with resonance decay and the directly freeze-out pions, respectively. In order to quantify the effect of the radial flow on the HBT radii, we apply the power-law fit to extract the parameters 

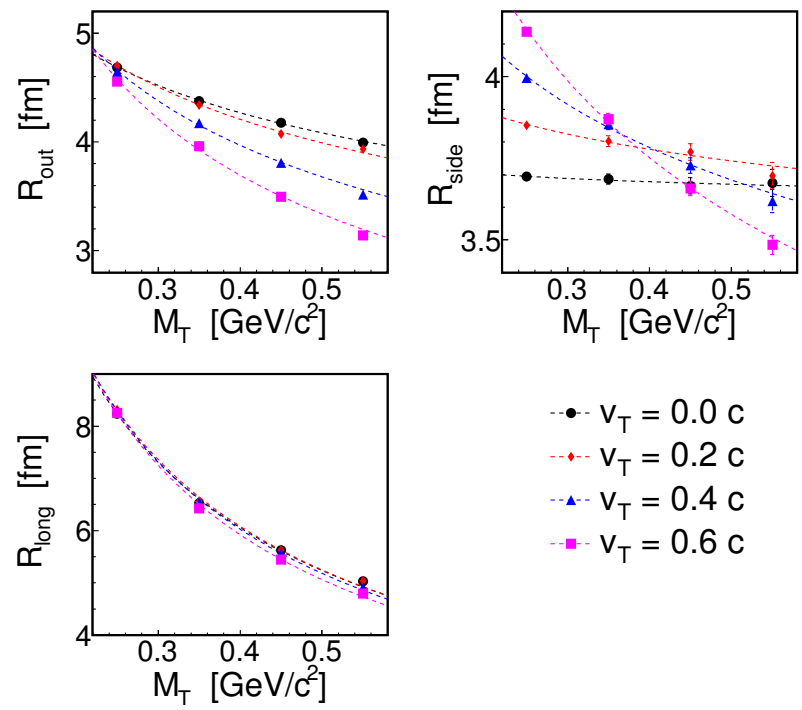

$$
\begin{aligned}
\cdots v_{T} & =0.0 c \\
\cdots v_{T} & =0.2 c \\
v_{T} & =0.4 c \\
v_{T} & =0.6 c
\end{aligned}
$$

Fig. 2. The HBT radii with different $v_{\mathrm{T}}$. Lines represent the fitted results using the power-law function $R_{i}\left(m_{\mathrm{T}}\right)=R_{i}^{\prime}\left(m_{\pi} / m_{\mathrm{T}}\right)^{\alpha}$.

$R^{\prime}$ and $\alpha$. The dependence of parameter $\alpha$ with increasing $v_{\mathrm{T}}$ can reflect the effect of the radial flow on the transverse momentum dependence of the HBT radii. Compared with the results of the pions with resonance decay, $R^{\prime}$ of the directly freeze-out pions does not depend on $v_{\mathrm{T}}$, which indicates that the radial flow affects only the drop of the HBT radii on the transverse momentum and does not change the value of the radii for the directly freezeout pions. The rise of $\alpha$ with increasing $v_{\mathrm{T}}$ for $R_{\text {out }}$ is faster than that for $R_{\text {side }}$ which suggests that $R_{\text {out }}$ is more susceptible to the radial flow. $R_{\text {out }}$ contains the information on space and time which leads $R_{\text {out }}$ to be more
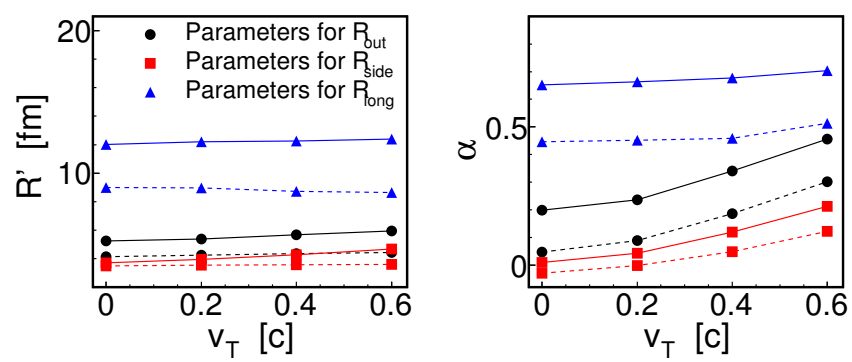

Fig. 3. Parameters $R^{\prime}$ in the left panel and $\alpha$ in the right panel as a function of $v_{\mathrm{T}}$ are extracted by the power-law fits from the HBT radii, where solid lines and dashed lines show the results of the directly freeze-out pions and the pions with resonance decay, respectively. 
susceptible than $R_{\text {side }}$ and $R_{\text {long }}$. It is necessary to mention that $\alpha$ is almost constant for the radii $R_{\text {long }}$, because the radial flow does not affect the longitudinal radii. The drop of $R_{\text {long }}$ is mainly caused by the longitudinal flow and the longitudinal direction of the source is satisfied with the boostinvariant in the THERMINATOR model [11, 17].

Figure 4 shows the HBT radii from the directly freeze-out pions and the pions with resonance decay for different $v_{\mathrm{T}}$. The values of $v_{\mathrm{T}}$ have different effects on the transverse momentum dependence of HBT radii with or without pions decayed from resonance. As $v_{\mathrm{T}}=0, R_{\text {out }}$ from the directly freeze-
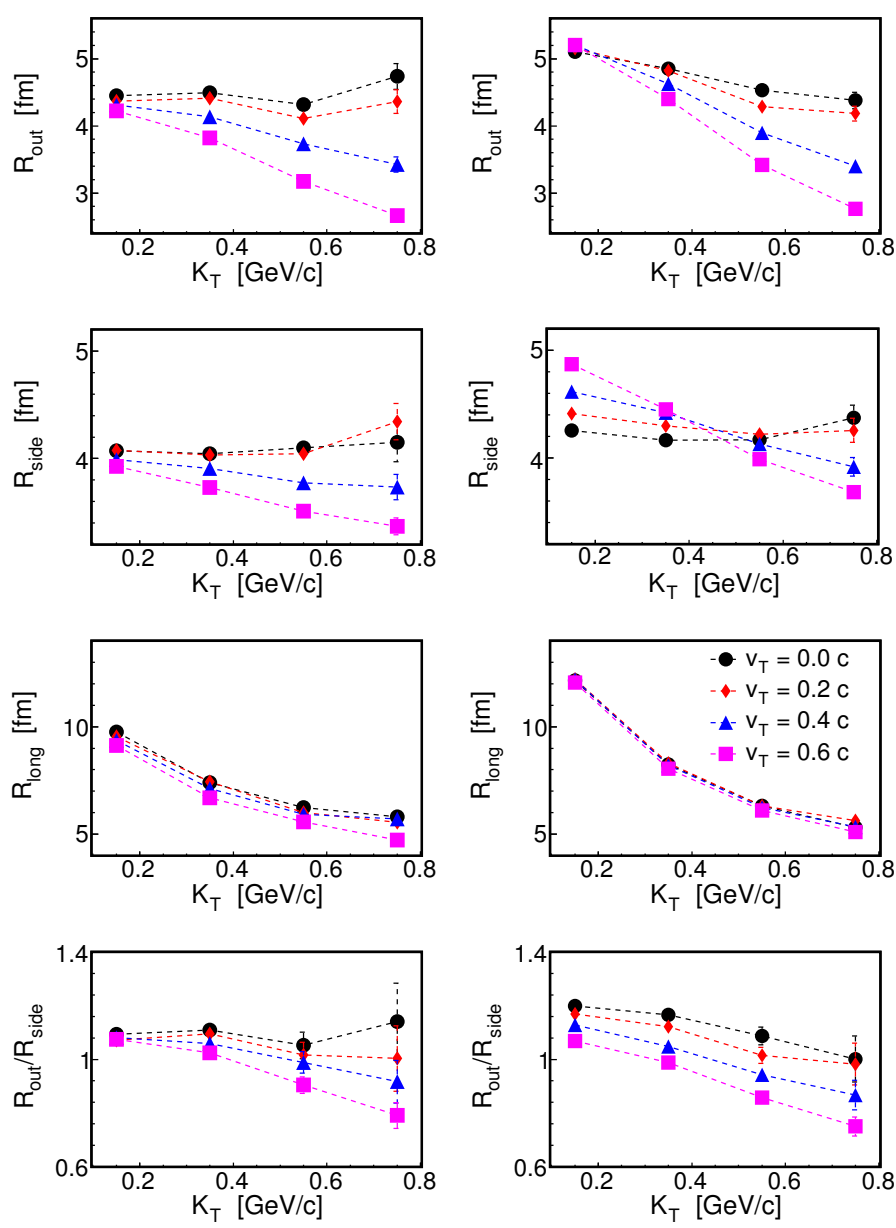

Fig. 4. The dependence of the HBT radii $R_{\text {out }}, R_{\text {side }}, R_{\text {long }}$ on transverse momentum of the pion pair is extracted from the directly freeze-out pions (left panel) and the pions with resonance decay (right panel) for different $v_{\mathrm{T}}$. The model parameters are as follows: $T=165.6 \mathrm{MeV}, \mu_{\mathrm{B}}=28.5 \mathrm{MeV}, \tau=9.91 \mathrm{fm}, \rho=7.43 \mathrm{fm}$. 
out pions stays constant with the increasing transverse momentum, but the drop of $R_{\text {out }}$ appears for the pions with resonance decay. The stronger radial flow makes the drop of $R_{\text {out }}$ more pronounced with increasing $K_{\mathrm{T}}$. As $v_{\mathrm{T}}=0, R_{\text {side }}$ stays constant with increasing $K_{\mathrm{T}}$ with or without pions decayed from resonance. With increasing $v_{\mathrm{T}}$, the radial flow reduces the value of $R_{\text {side }}$ from the directly freeze-out pions, but increases $R_{\text {side }}$ with resonance decays. The larger $v_{\mathrm{T}}$ makes the drop of $R_{\text {out }}$ with increasing $K_{\mathrm{T}}$ more pronounced than that of $R_{\text {side }}$ and reduces the ratio of $R_{\text {out }} / R_{\text {side }}$. The drop of $R_{\text {out }} / R_{\text {side }}$ with increasing $v_{\mathrm{T}}$ from pions with resonance decay is more obvious than that from the directly freeze-out pions.

Figure 5 shows the dependence of the HBT radii $R_{\text {out }}, R_{\text {side }}$ and the ratio of $R_{\text {out }} / R_{\text {side }}$ on the transverse momentum for the directly freeze-out pions, the pions decayed from resonance and the pions with resonance decay which include both all. As $v_{\mathrm{T}}=0, R_{\text {out }}$ and $R_{\text {side }}$ from the directly freeze-out pions stays constant, but $R_{\text {out }}$ of the pions decayed from resonance reduces with increasing $K_{\mathrm{T}}$ which leads to the drop of $R_{\text {out }}$ on $K_{\mathrm{T}}$ for the pions
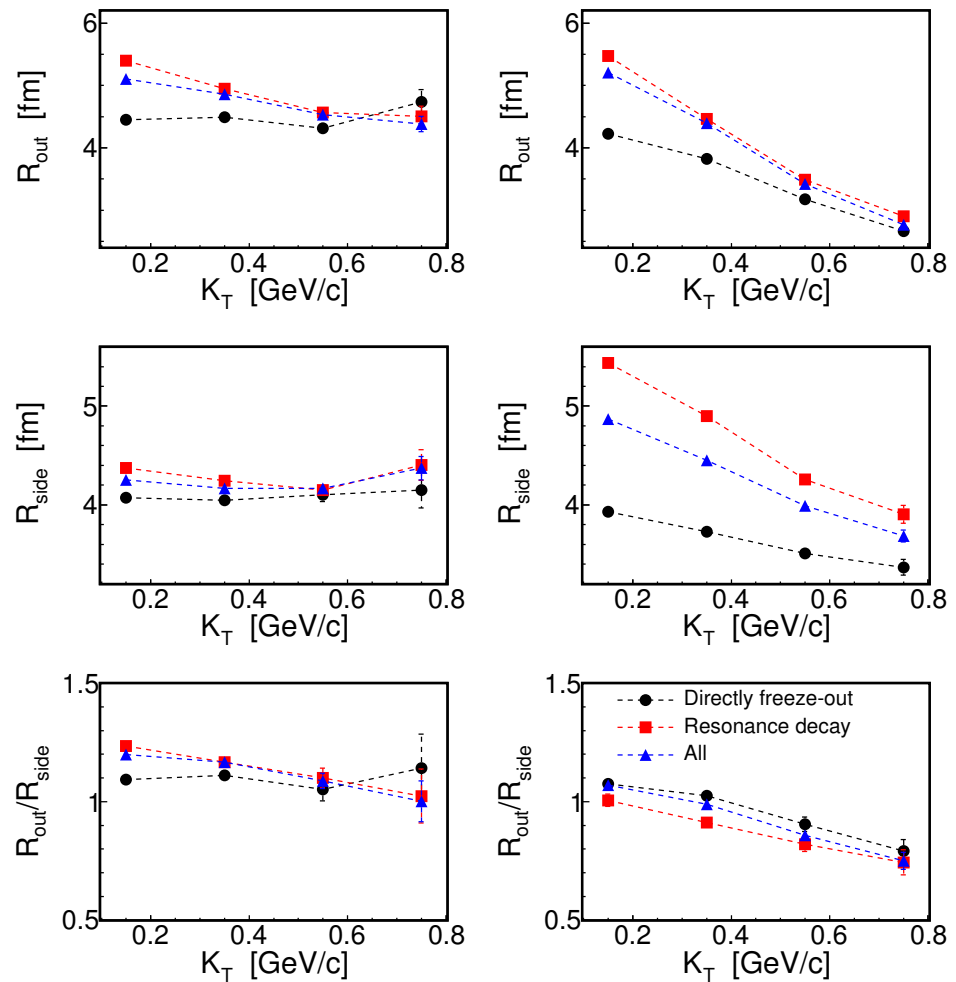

Fig. 5 . The dependence of the HBT radii $R_{\text {out }}, R_{\text {side }}$ and the ratio of $R_{\text {out }} / R_{\text {side }}$ on transverse momentum with and without resonance decays. Left panel is for $v_{\mathrm{T}}=0 c$, and right panel is for $v_{\mathrm{T}}=0.6 c$. 
with resonance decay. $R_{\text {out }}$ and $R_{\text {side }}$ decrease as $K_{\mathrm{T}}$ increases at $v_{\mathrm{T}}=0.6 \mathrm{c}$ for the directly freeze-out pions and the pions decayed from resonance, but the values of the radii for the pions decayed from resonance are much larger than those for the directly freeze-out pions.

Figures 6 and 7 show the dependence of the mean radial expansion flow value $\left\langle v_{\mathrm{T}}\right\rangle$, the mean cosine value of angle between freeze-out momentum and the radial direction $\langle\cos (\theta)\rangle,\left\langle x_{\text {out }}\right\rangle$ and $\left\langle x_{\text {side }}\right\rangle$ on the transverse momentum with $v_{\mathrm{T}}=0 c$ and $0.6 c$, where

$$
x_{\text {out }}=\frac{\left|\boldsymbol{r} \cdot \boldsymbol{p}_{\mathrm{T}}\right|}{\left|\boldsymbol{p}_{\mathrm{T}}\right|}, \quad x_{\text {side }}=\frac{\left|\boldsymbol{r} \times \boldsymbol{p}_{\mathrm{T}}\right|}{\left|\boldsymbol{p}_{\mathrm{T}}\right|} .
$$

As $v_{\mathrm{T}}=0$, the results of the directly freeze-out pions are quite different from those of the pions decayed from resonance. The values of $\left\langle v_{\mathrm{T}}\right\rangle,\langle\cos (\theta)\rangle$, $\left\langle x_{\text {out }}\right\rangle$ and $\left\langle x_{\text {side }}\right\rangle$ stay constant with increasing $K_{\mathrm{T}}$ for the pions which directly freeze out on the hypersurface. For the pions decayed from resonance, the dependence of those values on $K_{\mathrm{T}}$ is different. The values of $\left\langle v_{\mathrm{T}}\right\rangle$ and $\langle\cos (\theta)\rangle$ for the pions decayed from resonance are larger than those for the directly freeze-out pions with the $K_{\mathrm{T}}$ increase, which suggests the pions decayed from resonance have a different kind of collective motion along the radial direction, such as the space-momentum correlation, and increase the value of $\left\langle x_{\text {out }}\right\rangle$ and $\left\langle x_{\text {side }}\right\rangle$. As $v_{\mathrm{T}}=0.6 c$, the results of $\left\langle v_{\mathrm{T}}\right\rangle$ and $\langle\cos (\theta)\rangle$ for the directly freeze-out pions are similar to those for the pions decayed
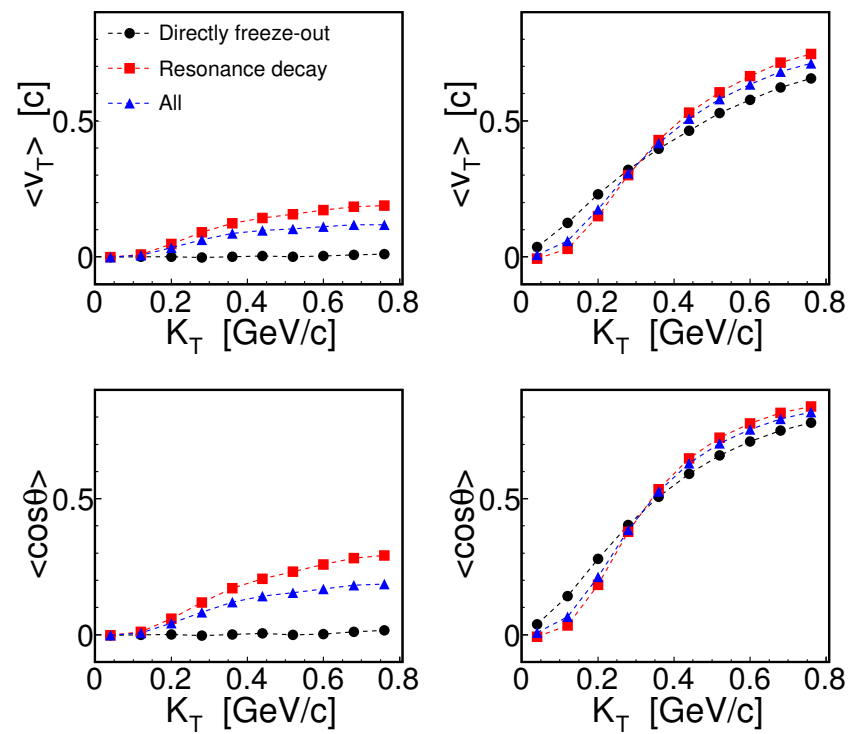

Fig. 6. The dependence of $\left\langle v_{\mathrm{T}}\right\rangle,\langle\cos (\theta)\rangle$ on transverse momentum with and without resonance decays. Left panel is for $v_{\mathrm{T}}=0 c$, and right panel is for $v_{\mathrm{T}}=0.6 c$. 

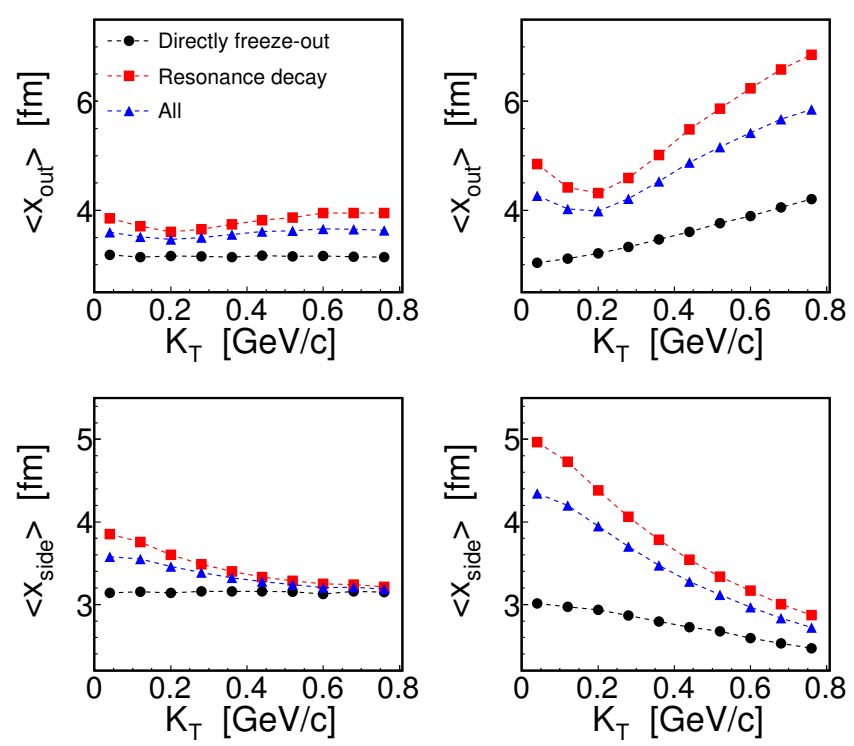

Fig. 7. The dependence of $\left\langle x_{\text {out }}\right\rangle,\left\langle x_{\text {side }}\right\rangle$ on transverse momentum with and without resonance decays. Left panel is for $v_{\mathrm{T}}=0 c$, and right panel is for $v_{\mathrm{T}}=0.6 c$.

from resonance, which suggests the effect of the space-momentum correlation is weaker than that of the radial flow. $\left\langle x_{\text {out }}\right\rangle$ and $\left\langle x_{\text {side }}\right\rangle$ of the pions decayed from resonance are much larger than those of the directly freeze-out pions, which indicates the space size of the source in the radial direction for the pions decayed from resonance is more sensitive to the increase of the radial flow. The pions decayed from resonance increase the HBT radii and strengthen the negative dependence of the radii on transverse momentum.

\section{Summary}

Based on the Blast-Wave parameterized model in THERMINATOR 2, we calculate the HBT parameters with different transverse velocities for $\mathrm{Au}+\mathrm{Au}$ central collisions at the RHIC energy $\sqrt{s_{N N}}=200 \mathrm{GeV}$ and study the effect of the radial flow and resonance decay on the HBT radii. The radial flow reduces the value of radii $R_{\text {out }}$ and increases the value of radii $R_{\text {side. }}$. The drop of the radii $R_{\text {out }}$ and $R_{\text {side }}$ decrease rapidly with increasing $v_{\mathrm{T}}$. The rise of $\alpha$ with increasing $v_{\mathrm{T}}$ for $R_{\text {out }}$ is faster than that for $R_{\text {side }}$ which suggests that $R_{\text {out }}$ is more susceptible to the radial flow. The larger $v_{\mathrm{T}}$ makes the drop of $R_{\text {out }}$ with increasing $K_{\mathrm{T}}$ more pronounced than that of $R_{\text {side }}$ and reduces the ratio of $R_{\text {out }} / R_{\text {side }}$. The pions decayed from resonance have a different kind of collective motion along the radial direction, such as 
the space-momentum correlation, and increase the size of the source in the radial direction, which increase the $\mathrm{HBT}$ radii and strengthen the negative dependence of the radii on transverse momentum.

This work was supported by the National Natural Science Foundation of China (NSFC Grant No. 11647148) and the Science and Technology Project of Jiangxi Provincial Education Department (No. GJJ160663).

\section{REFERENCES}

[1] F. Retiere, M.A. Lisa, Phys. Rev. C 70, 044907 (2004).

[2] G. Graef, M. Bleicher, M. Lisa, Phys. Rev. C 89, 014903 (2014).

[3] M.A. Lisa et al., Annu. Rev. Nucl. Part. Sci. 55, 357 (2005).

[4] C.J. Plumberg, C. Shen, U. Heinz, Phys. Rev. C 88, 069901 (2013).

[5] P. Bożek, Phys. Rev. C 90, 064913 (2014).

[6] J.B. Zhang et al., Chin. Phys. Lett 18, 1568 (2001).

[7] Y. Zhang et al., Phys. Rev. C 92, 014909 (2015).

[8] A. Kisiel, W. Florkowski, W. Broniowski, J. Pluta, Phys. Rev. C 73, 064902 (2006).

[9] Y. Zhang et al., J. Phys. G: Nucl. Part. Phys. 41, 125106 (2014).

[10] W. Florkowski, W. Broniowski, A. Kisiel, J. Pluta, Acta Phys. Pol. B 37, 3381 (2006).

[11] M. Chojnacki, A. Kisiel, W. Florkowski, W. Broniowski, Comput. Phys. Commun. 183, 746 (2011).

[12] S. Pratt et al., Nucl. Phys. A 566, 103c (1994).

[13] S.S. Adler et al. [PHENIX Collaboration], Phys. Rev. Lett. 93, 152302 (2004).

[14] Y. Zhang et al., J. Phys. G: Nucl. Part. Phys. 41, 025101 (2014).

[15] A. Adare et al. [PHENIX Collaboration], Phys. Rev. Lett. 112, 222301 (2014).

[16] T. Csörgö, B. Lörstad, Phys. Rev. C 54, 1390 (1996).

[17] L. Adamczyk et al. [STAR Collaboration], Phys. Rev. C 88, 034906 (2013). 